\title{
Peritoneal carcinosis in male germ cell tumor patients: a registry study compiled by the German Testicular Cancer Study Group (GTCSG)
}

\author{
Christoph Seidel ${ }^{1} \cdot$ Marcus Hentrich $^{2}$ - Stefanie Zschäbitz ${ }^{3}$ Pia Paffenholz ${ }^{4}$. Axel Heidenreich ${ }^{4} \cdot$ Tim Nestler $^{5}$. \\ Ben $\operatorname{Tran}^{6}$. Stefanie Fischer ${ }^{7}$. Gedske Daugaard ${ }^{8}$. Sebastian Ochsenreither ${ }^{9,10}$. Margarida Brito ${ }^{11}$. \\ Friedemann Zengerling ${ }^{12} \cdot$ Constantin Schwab ${ }^{13} \cdot$ Carsten Bokemeyer $^{1} \cdot$ Christoph Oing $^{1,14}$ (1)
}

Received: 26 May 2021 / Accepted: 11 November 2021 / Published online: 7 January 2022

(c) The Author(s) 2022

\begin{abstract}
Purpose To report on the clinical characteristics, outcome, and frequency of peritoneal carcinosis (PC) in patients with advanced germ cell tumors (GCT), a multicenter registry analysis was carried out.

Methods A multicenter registry analysis was conducted by the German Testicular Cancer Study Group (GTCSG) with international collaborators. Data was collected and analyzed retrospectively. Patients were eligible for inclusion if PC was diagnosed either by radiologic or histopathologic finding during the course of disease. Descriptive and explorative statistical analysis was carried out with cancer-specific survival (CSS) as primary study endpoint.

Results Collaborators from ten GCT expert centers identified 28 GCT $(0.77 \%)$ patients with PC after screening approximately 3767 GCT patient files and one case was contributed from a cancer registry request. Patients were diagnosed from 1997 to 2019 at a median age of 37 years (interquartile range, 13). Two patients (7\%) presented with stage I and 27 patients (93\%) with synchronous metastatic disease at first diagnosis. The primary histology was seminoma in seven (27\%) and nonseminoma in 21 patients (72\%). PC was detected after a median of 15.3 months from primary diagnosis (range 0-177) and two consecutive treatment lines (range 0-5), respectively. The median CSS from the time of detection of PC was 10.5 months (95\% Confidence Interval 0.47-1.30) associated with an overall 2-year CSS rate of 30\%.

Conclusion PC represents a rare tumor manifestation in GCT patients and was primarily associated with the occurrence of advanced cisplatin-refractory disease conferring to a dismal prognosis.
\end{abstract}

Keywords Testicular cancer $\cdot$ Germ cell tumor $\cdot$ Peritoneal carcinosis

\section{Introduction}

Testicular germ cell tumors (GCTs) are the most common solid organ malignancy among young men aged between 15 and 35 years [1]. Due to an excellent sensitivity towards cisplatin-based chemotherapy as a part of multimodal treatment approaches, advanced GCTs represent a curable malignant disease associated with 5-year survival rates ranging from 50 up to $96 \%$ in advanced disease stages [2-4]. Metastatic dissemination commonly involves the retroperitoneal lymph nodes, lymph nodes of the mediastinum, and lungs. Other

Carsten Bokemeyer and Christoph Oing contributed equally.

Christoph Oing

c.oing@uke.de

Extended author information available on the last page of the article metastatic sites may include liver, bone and brain which are less common and associated with adverse outcomes [5-7]. Until now, peritoneal carcinosis (PC) in GCT patients was described by case reports and small case series only, revealing merely little data concerning the frequency, potential causes of development and impact on the patient's outcome [8-12]. The largest series of cases published so far, comprised the course of disease of five GCT patients with PC from a French high-volume center. As four of the patients received retroperitoneal lymph node dissection (RPLND) prior to the detection of PC, the authors hypothesized that RPLND may have caused a route of tumor extension from a lymphatic leakage during surgery which promoted consecutive PC development [8]. This hypothesis was, furthermore, shared by two other case reports, presenting single patient cases with GCT and PC development after RPLND or lymph node biopsy $[9,10]$. Another case report, however, depicted 
the course of disease of a heavily pre-treated patient who developed PC after multiple, partially inadequately dosed treatment lines without receiving prior RPLND. Here, Abe et al. concluded that multiple treatment regimens that were applied could have increased the aggressiveness of tumor biology, associated with development of a chemo-resistant phenotype resulting in the development of PC at late-stage disease [12].

To report on the clinical characteristics, outcome, and frequency of PC in GCT patients, a multicenter registry analysis was carried out. It was also the aim to investigate the potential correlation between the occurrence of PC and prior RPLND as a route of dissemination.

\section{Patients and methods}

\section{Study population and inclusion criteria}

This multicenter registry analysis was conducted by the German Testicular Cancer Study Group (GTCSG) in association with international collaborators. Clinical information was collected retrospectively via pseudonymized case report forms (CRFs) from the medical records. CRFs were subsequently centrally stored and assessed at the University Medical Center Hamburg-Eppendorf. A total of 29 cases from eleven participating centers were collected and eligible for analysis. GCT patients were eligible for the study, if PC was detected by histological or radiologic examination any time during the course of the disease. Only male GCT patients were considered for analysis.

The study was approved by the local ethics committee of the Chamber of Physicians Hamburg (File Number: PV7058).

\section{Statistical analysis}

The objective of this multicenter registry study was to provide data concerning the clinical characteristics, outcome, and frequency of PC in GCT patients. It was also the aim to investigate the potential correlation between the occurrence of PC and prior RPLND as a route of dissemination. To find prognostic factors patient characteristics were correlated with the outcome.

The cancer-specific survival (CSS) defined as the time from detection of PC until to the date of death from GCT disease was considered as primary study endpoint. Patients lost to follow-up were censored at the date of last visit. Survival analysis was conducted using the method of Kaplan-Meier [13] and log-rank test was applied to compare survival rates. The following patient characteristics were tested as potential prognostic factors: seminoma vs. non-seminoma, late recurrence after first line systemic treatment vs. early recurrence after first line systemic treatment, gonadal disease vs. extragonadal disease, IGCCCG good vs. intermediate vs. poor, local treatment of $\mathrm{PC}$ vs. no local treatment of PC, and synchronous metastatic disease vs. metachronous metastatic disease. A two-sided $p$ value $<0.05$ was considered statistically significant. Statistical analyses were conducted using statistical package for the social science version 18 (SPSS). Due to the low patient number a multivariate regression model was not conducted.

\section{Results}

\section{Patient characteristics}

Eleven participating institutions identified a total of 29 GCT patients with PC. Patients were first diagnosed with GCT from 1997 to 2019 at a mean age of 37 years at first diagnosis (range 19-60 years).The primary histology at first diagnosis was pure seminoma in seven (27\%) and nonseminoma in $22(72 \%)$ patients, respectively. Two patients (7\%) were first diagnosed with stage I disease according to UICC [14] and 27 patients (93\%) presented with synchronous metastatic disease at the time of their diagnosis with stage II $(n=9)$ and III $(n=18)$ disease, respectively. Patients with synchronous metastatic disease were classified as poor prognosis in $16(55 \%)$, intermediate prognosis in six (21\%), and good prognosis in seven (24\%) patients according to the IGCCCG criteria [2]. Patient characteristics are described in detail in Table 1.

\section{Course of disease and treatment}

The patients of this cohort received a median of three different systemic treatment lines from the timepoint of first diagnosis (range 1-7). Recurrence after first line systemic treatment was reported in 27 patients $(93 \%)$ and two patients (7\%) died during or prior to first line systemic treatment due to tumor progression and pneumonia (7\%). Of 28 patients treated with first line systemic treatment 24 patients (86\%) received further salvage systemic therapies. The most frequently applied salvage regimen was high dose carboplatin with etoposide and autologous peripheral stem cell transplantation (PBSCT) administered in eight patients followed by paclitaxel, ifosfamide and cisplatin (TIP) administered in seven patients. Of thirteen patients receiving consecutive third line treatment, gemcitabine and oxaliplatin was administered to ten patients. Details concerning the different chemotherapy lines administered are reported in Table 1 . Surgical procedures which included the resection of metastases of the peritoneum were carried out in seven patients during their course of disease (24\%) Additional treatment 
Table 1 Patient characteristics

\begin{tabular}{|c|c|c|}
\hline Characteristics & $\begin{array}{l}\text { Absolute number of patients } \\
n=29\end{array}$ & $\%$ \\
\hline Median age (years) & 37 & (range 18-60) \\
\hline \multicolumn{3}{|l|}{ Histology } \\
\hline Seminoma & 7 & $24 \%$ \\
\hline Non-Seminoma & 22 & $76 \%$ \\
\hline \multicolumn{3}{|l|}{ UICC stage at primary diagnosis } \\
\hline UICC I & 2 & $7 \%$ \\
\hline UICC Stage II & 9 & $31 \%$ \\
\hline UICC Stage III & 18 & $62 \%$ \\
\hline \multicolumn{3}{|c|}{ IGCCCG classification at primary diagnosis of stage II/III disease } \\
\hline Good & 7 & $24 \%$ \\
\hline Intermediate & 6 & $21 \%$ \\
\hline Poor & 16 & $55 \%$ \\
\hline \multicolumn{3}{|l|}{ Primary site of the tumor } \\
\hline Gonadal & 23 & $79 \%$ \\
\hline Extragonadal retroperitoneal & 6 & $21 \%$ \\
\hline Median number of treatment lines & 3 & (range $1-7$ ) \\
\hline Salvage therapy performed & 19 & $66 \%$ \\
\hline High dose chemotherapy performed & 13 & $45 \%$ \\
\hline First line systemic treatment & 28 & $97 \%$ \\
\hline BEP & 18 & $62 \%$ \\
\hline VIP & 4 & $14 \%$ \\
\hline $\mathrm{EP}$ & 3 & $10 \%$ \\
\hline TIP & 1 & $3 \%$ \\
\hline VIC & 1 & $3 \%$ \\
\hline HD-VIP & 1 & $3 \%$ \\
\hline Second line systemic treatment & 24 & $83 \%$ \\
\hline $\mathrm{HD}-\mathrm{CE}$ & 8 & $28 \%$ \\
\hline TIP & 7 & $24 \%$ \\
\hline GO & 4 & $14 \%$ \\
\hline Cisplatin or Carboplatin + Etoposid & 2 & $3 \%$ \\
\hline HD-VIP & 2 & $7 \%$ \\
\hline CGP & 1 & $3 \%$ \\
\hline Third line systemic treatment & 13 & $45 \%$ \\
\hline $\mathrm{ACO}$ & 1 & $3 \%$ \\
\hline TIP & 1 & $3 \%$ \\
\hline $\mathrm{GO}(\mathrm{P} / \mathrm{I})$ & 10 & $34 \%$ \\
\hline $\mathrm{EP}$ & 1 & $3 \%$ \\
\hline HD-CE & 1 & $3 \%$ \\
\hline \multicolumn{3}{|c|}{ Resection of metastases after first line treatment } \\
\hline RPLND & 13 & $45 \%$ \\
\hline Atypical lung resection & 1 & $2 \%$ \\
\hline Removal of peritoneal lesions & 1 & $2 \%$ \\
\hline
\end{tabular}

BEP Bleomycin, Etoposide, Cisplatin; EP Etoposide, Cisplatin; VIP Etoposide, Ifosfamide, Cisplatin; TIP Paclitaxel, Ifosfamide, Cisplatin; VIC Vindesin, Ifosfamide, Carboplatin; HD VIP High dose Etoposide, Ifosfamide, Cisplatin; GO Gemcitabine, Oxaliplatin; $C G P$ Carboplatin, Gemcitabine, Paclitaxel; $A C O$ Adriamycin, Cyclophosphamide, Vincristin 
with Hyperthermic Intra-Peritoneal Chemotherapy (HIPEC) was administered to five patients (17\%).

\section{Frequency and detection of PC}

Altogether 28 cases were detected from ten centers after screening their databases which included approximately 3767 GCT patient files which corresponds to a prevalence of $0.74 \%$. One additional case of PC was detected after the search from a local cancer registry. All participating centers were tertiary center hospitals and experienced in GCT patient care. Two participating institutions had exclusively access to patients with metastatic disease. PC diagnosis was confirmed with biopsy or surgical resection in six $(29 \%)$ or by radiological examination in 23 cases (79\%). Here histopathological evaluation revealed seminoma in three cases and choriocarcinoma, immature teratoma, and teratoma with adenocarcinoma and spindle cell malignant tumor in one case each. Results from the histological examination of the specimens if biopsy or surgical resections were carried out are depicted in detail in Table 1. Figure 1A illustrates an exemplar of the pathological examination of a resected PC lesion demonstrating immature teratoma and Fig. 1B displays carcinoma cells detected in ascites after paracenteses was carried out. Figure 1C shows the radiological finding of a patient from this cohort with PC lesions compromising intestinal loops leading to bowel obstruction.

\section{Development and symptoms of PC}

PC was diagnosed after a median of 15.3 (95\% CI 18.8-79.5) months from primary diagnosis and after a median of two consecutive lines of platinum-based chemotherapy, respectively (range 0-5). Clinical symptoms associated with PC were described in 15 of 29 cases (52\%). In two patients (7\%) data concerning the occurrence of symptoms was not specified by the investigators and concerning 12 patients (41\%) no PC-specific symptoms were documented. The most frequent symptoms associated with PC were abdominal pain in seven (24\%), ascites in five (17\%) and ileus in three patients (10\%). Further symptoms described are reported in Table 1. Paracenteses was carried out in six patients (21\%) associated with a proof of malignant germ cell tumor cells by cytological examination in three patients $(10 \%)$. Prior to the detection of PC, 13 patients (45\%) received RPLND.

\section{Patient outcomes}

At the time of data acquisition nine patients (28\%) were still alive and 20 patients (69\%) had succumbed to their disease associated. The median CSS from PC diagnosis was 10.5 months (range 0.30-104.22) associated with a 2-year CSS rate of $30 \%$ (Fig. 2). Concerning patient characteristics with a potential impact on CSS univariate statistical analysis revealed no patient characteristics that significantly correlated with CSS (Table 2).
Fig. 1 A Picture of immature teratoma, consisting of densely aggregated, nodular, atypical, small-to-medium-sized cell clusters (Coutesy Dr. Schwab, Heidelberg). B Cytological analysis of the ascitic fluid. May-Grünwald-Giemsa staining showed atypical pleomorphic cells with enlarged nuclei, dispersed chromatin and cytoplasmatic vacuoles, arranged as loosely cohesive clusters (Courtesy Dr. Heinz Diem, Munich). C CT scan schowing signs of peritoneal carcinosis (arrows) in a 26-year-old male with metastatic non-seminoma compromising intestinal loops leading to bowel obstruction

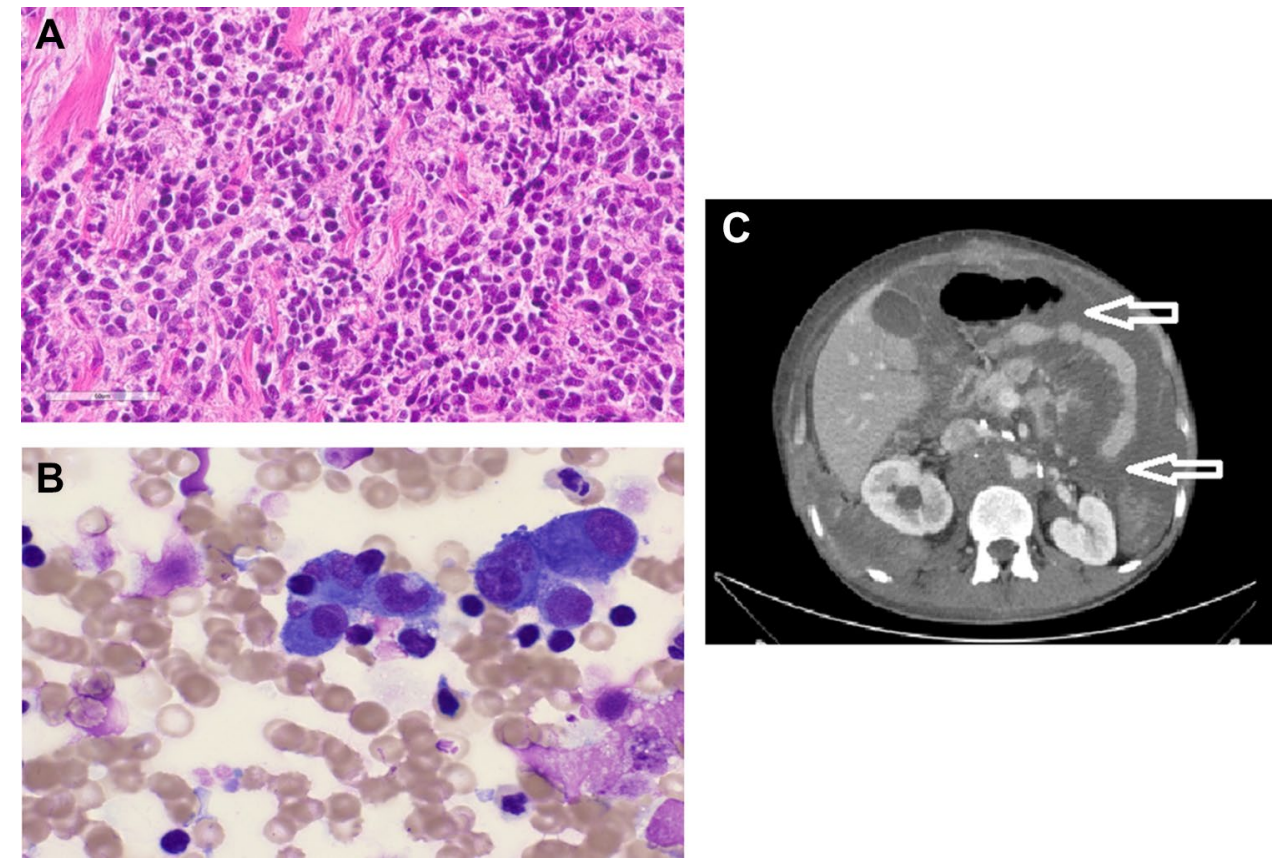


Fig. 2 Cancer-specific survival in years from first diagnosis of peritoneal carcinosis
Table 2 Results of univariate analysis concerning CSS

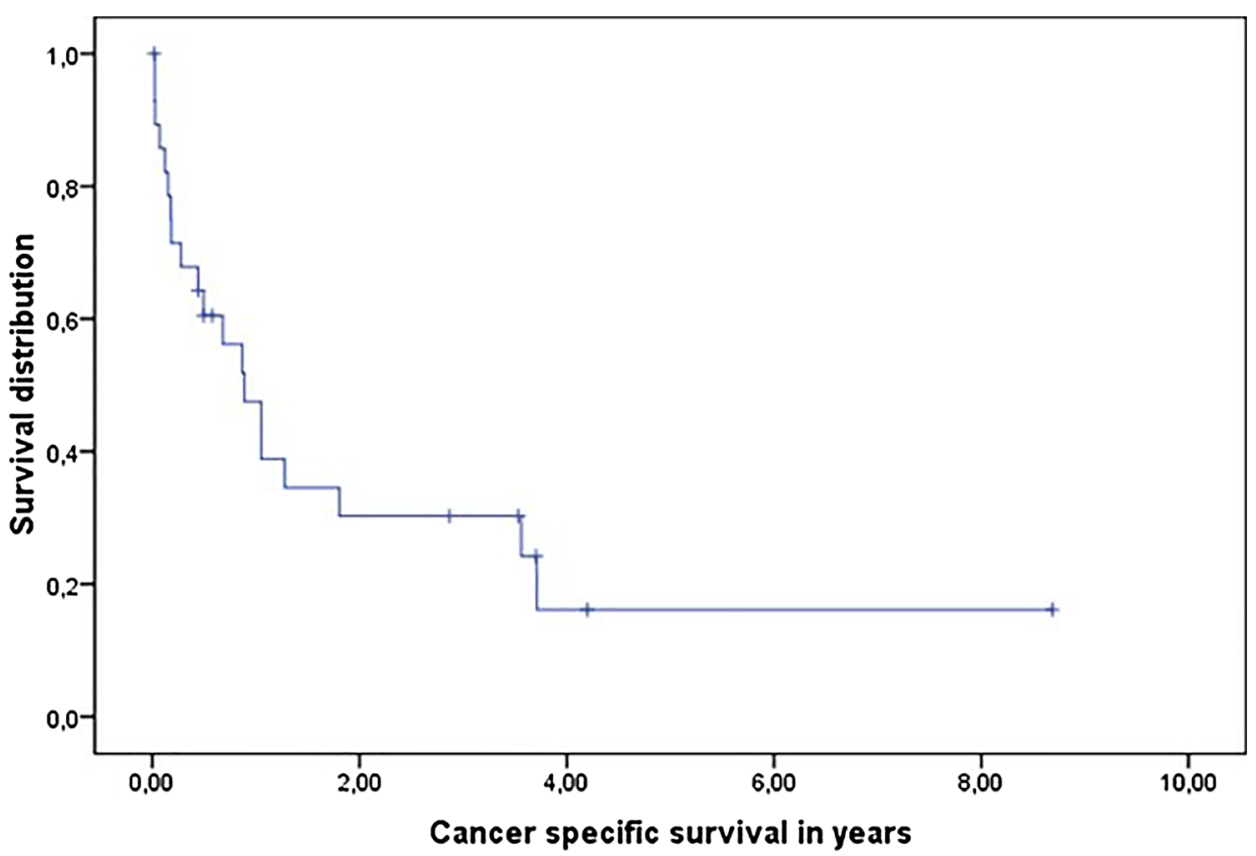

\begin{tabular}{lll}
\hline Factor & 2-year CSS rate & $p$ value \\
\hline Seminoma vs. Non-Seminoma & $29 \%$ vs. $30 \%$ & 0.807 \\
Gonadal vs. Extragonadal & $34 \%$ vs. $16 \%$ & 0.704 \\
Metachronous vs. Synchronous metastatic disease & $100 \%$ vs. $25 \%$ & 0.514 \\
IGCCCG good vs. intermediate vs. poor & $33 \%$ vs. $29 \%$ vs. 24\% & 0.692 \\
Local PC treatment yes vs. no & $22 \%$ vs. $16 \%$ & 0.305 \\
\hline
\end{tabular}

\section{Discussion}

PC is a very rare condition in GCT patients and to date only single case reports and a small series of five patients addressing this condition are available. The aim of this study was to present sufficient data to adequately describe the patient characteristics, outcome, approximate frequency and potential causes concerning the route of dissemination of PC in GCT patients.

With a multicentric registry complied by the German Testicular Cancer Study Group (GTCSG) 28 GCT patients with PC were detected after screening approximately 3,767 patients, while one additional case was contributed from a regional cancer registry request. With a total of 29 cases this is so far the largest report on PC in GCT. With an approximate prevalence of $0.77 \%$, our results confirm the observation by Andre et al. that the occurrence of PC from GCT is very rare but not exceptional. As earlier reports postulated a correlation between the development of PC and prior RPLND or lymph node biopsy [8-10] our analysis revealed that only $45 \%$ of patients received RPLND prior to the detection of PC. RPLND, therefore, seems not to be the only driver of PC development. In contrast, our cohort clearly depicts patients with adverse clinical characteristics, such as synchronous metastatic disease, poor prognosis according IGCCCG, and an exceptional high rate of cisplatin-refractory disease. Here $83 \%$ of our patients underwent at least two different treatment lines, while two patients died during or prior to first line systemic treatment. An overall 2-year CSS rate of $30 \%$ underlines this observation and is in line with previous reports on patients failing two or more treatment lines $[15,16]$. We, therefore, hypothesize that PC may be in most of the cases the result of aggressive, multiply relapsed and thus treatment-refractory tumors, associated with dismal prognosis.

Despite the fact that this presented series is the largest analysis of PC in GCT patients, so far, major limitations include the retrospective study design and the still limited number of cases available highlighting the rarity of PC in GCT. Furthermore, only tertiary specialised GCT centers participated and two participating centres only had access to patient files with disseminated disease only which will probably lead to a higher incidence of PC. 
In conclusion, peritoneal carcinosis is a rare phenomenon occurring in advanced and mostly heavily pretreated germ cell tumor patients who generally face a dismal prognosis.

Author contributions CS: protocol/project development, data collection and management, data analysis, manuscript writing/editing. MH, SZ, PP, AH, TN, BT, SF, GD, SO, MB, CS, FZ: data collection, manuscript editing. $\mathrm{CB}$ : protocol/project development, data analysis, manuscript editing. CO: protocol/project development, data collection and management, data analysis, manuscript writing/editing.

Funding Open Access funding enabled and organized by Projekt DEAL.

\section{Declarations}

Conflict of interest All authors declare no conflict of interest.

Research involving human participants and/or animals This research did not involve any study related treatment of human participants or animals.

Informed consent All patients had given written informed consent for the use of data regarding their disease characteristics, treatment and outcomes within research projects.

Open Access This article is licensed under a Creative Commons Attribution 4.0 International License, which permits use, sharing, adaptation, distribution and reproduction in any medium or format, as long as you give appropriate credit to the original author(s) and the source, provide a link to the Creative Commons licence, and indicate if changes were made. The images or other third party material in this article are included in the article's Creative Commons licence, unless indicated otherwise in a credit line to the material. If material is not included in the article's Creative Commons licence and your intended use is not permitted by statutory regulation or exceeds the permitted use, you will need to obtain permission directly from the copyright holder. To view a copy of this licence, visit http://creativecommons.org/licenses/by/4.0/.

\section{References}

1. Huyghe E, Matsuda T, Thonneau P (2003) Increasing incidence of testicular cancer worldwide: a review. J Urol 170:5-11

2. IGCCCG (1997) International Germ Cell Consensus Classification: a prognostic factor-based staging system for metastatic germ cell cancers. International Germ Cell Cancer Collaborative Group. J Clin Oncol 15:594-603

3. Beyer J, Collette L, Sauvé N et al (2021) International germ cell cancer classification update consortium survival and new prognosticators in metastatic seminoma: results from the IGCCCG-Update Consortium. J Clin Oncol. https://doi.org/10.1200/ JCO.20.03292

4. Hanna NH, Einhorn LH (2014) Testicular cancer-discoveries and updates. N Engl J Med 20(371):2005-2016

5. Patel HD, Singla N, Ghandour RA et al (2019) Site of extranodal metastasis impacts survival in patients with testicular germ cell tumors. Cancer 125:3947-3952. https://doi.org/10.1002/cncr. 32427 (Epub 2019 Jul 29)

6. Feldman DR, Lorch A, Kramar A et al (2016) Brain metastases in patients with germ cell tumors: prognostic factors and treatment options-an analysis from the global germ cell cancer group. J Clin Oncol 34:345-351

7. Oing C, Oechsle K, Necchi A et al (2017) Impact of primary metastatic bone disease in germ cell tumors: results of an International Global Germ Cell Tumor Collaborative Group G3 Registry Study. Ann Oncol 28:576-582 (PubMed: 27993806)

8. Andre F, Fizazi K, Culine S et al (2000) Peritoneal carcinomatosis in germ-cell tumor: relations with retroperitoneal lymph node dissection. Am J Clin Oncol 23:460-462. https://doi.org/10.1097/ 00000421-200010000-00006

9. Barakos JA, Brookjeffrey R Jr, McCaninch JW et al (1986) Computerized tomography diagnosis of diffuse intraperitoneal metastases after retroperitoneal lymphadenectomy for testicular carcinoma. J Urol 136:680-681

10. Baniel J, Foster RS, Rowland RG et al (1995) Complications of post-chemotherapy retroperitoneal lymph node dissection. J Urol 153:976-980

11. Heafield RJ, Mead CM, Tung KT (1994) Case report: intraperitoneal metastases from testicular germ cells tumors. Br J Radiol 67:501-503

12. Abe T, Shinohara N, Harabayashi T et al (2004) Peritoneal carcinomatosis in refractory seminoma. Int J Urol 11:184-186. https:// doi.org/10.1111/j.1442-2042.2003.00760.x

13. Kaplan EL, Meier P (1958) Nonparametric estimation from incomplete observations. J Am Stat Assoc 53:457-481. https:// doi.org/10.2307/2281868.JSTOR2281868

14. Sobin LH, Gospodarowicz MK, Wittekind C (2009) TNM classification of malignant tumors. UICC International Union Against Cancer, 7th edn. Wiley-Blackwell, New York

15. Oechsle K, Kollmannsberger C, Honecker F et al (2011) Longterm survival after treatment with gemcitabine and oxaliplatin with and without paclitaxel plus secondary surgery in patients with cisplatin-refractory and/or multiply relapsed germ cell tumors. German Testicular Cancer Study Group. Eur Urol 60:850-855. https://doi.org/10.1016/j.eururo.2011.06.019

16. Seidel C, Oechsle K, Lorch A et al (2016) Efficacy and safety of gemcitabine, oxaliplatin, and paclitaxel in cisplatin-refractory germ cell cancer in routine care-Registry data from an outcomes research project of the German Testicular Cancer Study Group. Urol Oncol 34:167.e21-8. https://doi.org/10.1016/j.urolonc.2015. 11.007

Publisher's Note Springer Nature remains neutral with regard to jurisdictional claims in published maps and institutional affiliations. 


\section{Authors and Affiliations}

\section{Christoph Seidel ${ }^{1} \cdot$ Marcus Hentrich $^{2} \cdot$ Stefanie Zschäbitz ${ }^{3} \cdot$ Pia Paffenholz ${ }^{4}$. Axel Heidenreich ${ }^{4} \cdot$ Tim Nestler $^{5}$. ${\text { Ben } \operatorname{Tran}^{6} \text {. Stefanie Fischer }}^{7}$. Gedske Daugaard ${ }^{8}$. Sebastian Ochsenreither ${ }^{9,10}$. Margarida Brito ${ }^{11}$. Friedemann Zengerling ${ }^{12} \cdot$ Constantin Schwab ${ }^{13} \cdot$ Carsten Bokemeyer $^{1} \cdot$ Christoph Oing $^{1,14}$ [C]}

1 Department of Oncology, Hematology and Bone Marrow Transplantation with Division of Pneumology, University Medical Center Hamburg-Eppendorf, Martinistraße 52, 20246 Hamburg, Germany

2 Department of Medicine III-Haematology/Oncology, Red Cross Hospital, Munich, Germany

3 Department of Medical Oncology, National Centre for Tumour Diseases, Heidelberg University Hospital, Heidelberg, Germany

4 Department of Urology, University Hospital Cologne, Cologne, Germany

5 Department of Urology, Federal Armed Services Hospital Koblenz, Koblenz, Germany

6 Peter MacCallum Cancer Centre, Melbourne, Australia

7 Department of Medical Oncology and Hematology, Cantonal Hospital St. Gallen, St. Gallen, Switzerland
8 Department of Oncology, Copenhagen University Hospital, Rigshospitalet, Copenhagen, Denmark

9 Department of Medical Oncology and Hematology, Charité Campus Benjamin Franklin, Berlin, Germany

10 Charité Comprehensive Cancer Center, Charité Campus Mitte, Berlin, Germany

11 Instituto Português de Oncologia de Lisboa, Lisboa, Portugal

12 Department of Urology, University Hospital Ulm, Ulm, Germany

13 Department of Pathology, National Centre for Tumour Diseases, Heidelberg University Hospital, Heidelberg, Germany

14 Mildred Scheel Cancer Career Center HaTriCs4, University Cancer Center Hamburg, University Medical Center Hamburg-Eppendorf, Hamburg, Germany 